\title{
Targeted treatment of advanced ovarian cancer: spotlight on rucaparib
}

This article was published in the following Dove Press journal:

Therapeutics and Clinical Risk Management

\section{Diana C Pearre \\ Krishnansu S Tewari \\ Division of Gynecologic Oncology, Department of Obstetrics \& Gynecology, University of California, Irvine Medical Center, Orange, CA, USA}

\begin{abstract}
The last 2 years have ushered in a new era in ovarian cancer therapy with the US Food and Drug Administration's (FDA) approval of poly-ADP ribose polymerase (PARP) inhibitors (PARPi). One of the deadliest cancers that women experience, ovarian cancer, is most often diagnosed in advanced stages. Although cytoreductive surgery and (platinum/taxane-based) chemotherapy can place the majority of patients into remission, most will experience a relapse of their disease in their lifetime. This has led to studies exploring the benefits and efficacy of maintenance treatment. This review will briefly discuss the history of maintenance therapy as well as focus on the FDA's approval of rucaparib and its companion tumor profiling test, in the US. It will describe how women with deleterious mutations in the $B R C A$ gene, through their inherent deficiency in homologous recombination, presented scientists with a target to exploit through a concept known as synthetic lethality. Not only did this lead to a targeted treatment for BRCA mutation carriers but for other patients with deficiencies in homologous recombination and, more broadly, also in platinum-sensitive patients. The focus of this review will be on rucaparib in the US, approved for both maintenance of platinum-sensitive recurrent ovarian cancer and treatment in the third-line setting and beyond. It has the broadest indication amongst the three PARPi in ovarian cancer. Furthermore, the ongoing trials using rucaparib in ovarian cancer and other disease types will be discussed.
\end{abstract}

Keywords: BRCA, PARP inhibitor, targeted therapy, rucaparib, ovarian cancer

\section{Introduction}

Ovarian cancer is the fifth leading cause of death in women in the US. According to the Center for Disease Control's Surveillance, Epidemiology, and End Results Program, there will be an estimated number of 22,240 new cases of ovarian cancer in the US in 2018. Although 1 in 73 women will develop ovarian cancer in their lifetime, this number is declining.

The mainstay of therapy in ovarian cancer focuses on a combination of both chemotherapy and surgery. The principal surgery is a major debulking or cytoreductive surgery (CRS) removing a patient's uterus, bilateral tubes and ovaries, omentum, pathologically enlarged lymph nodes, and peritoneal surfaces involving disease. This may also involve removal of portions of the small or large bowel and removing the involved areas of the diaphragm and the spleen. The principles of surgery for ovarian cancer involve attempting to perform the most complete cytoreductive surgery (CRS) possible for the patient - ultimately achieving no gross residual disease. In meta-analyses, optimal debulking has been associated with significantly improved overall survival (OS) and progression-free survival (PFS). ${ }^{2,3}$ Adjuvant therapy is recommended as per the National Comprehensive Cancer Network (NCCN) guidelines, even for early-stage disease if criteria are met. ${ }^{4}$ The concept of adding chemotherapy
Correspondence: Krishnansu S Tewa Division of Gynecologic Oncology, Department of Obstetrics \& Gynecology, University of California, Irvine Medical Center, 333 The City Boulevard West, Orange, CA 92868, USA

Tel + I 7l 44568020

Fax +I 7144566632

Email ktewari@uci.edu 
as neoadjuvant treatment to help achieve this surgical goal of removing all grossly visible disease at the time of CRS is also done in various situations. ${ }^{5-7}$ In the setting of recurrent ovarian cancer, a patient is considered platinum-sensitive if there is at least a 6-month interval between the completion of chemotherapy and disease recurrence. In this setting they tend to receive a platinum-based doublet for their recurrent disease, based on evidence from large randomized controlled trials (RCTs) that this is beneficial. ${ }^{8,9}$ If disease recurs prior to that 6-month interval, they are considered platinumresistant and may be prescribed a regimen combined with bevacizumab as shown in the AURELIA trial. ${ }^{10}$

More studies have shown that patients with a genetic predisposition to breast and ovarian cancer, specifically with a mutation in $B R C A 1$ or $B R C A 2$, tend to respond to therapy better. ${ }^{11,12}$ In the wild-type setting, BRCA proteins repair double-strand DNA breaks through homologous recombination. Patients with germline BRCA mutations, as well as those whose tumors have somatic mutations in BRCA, are unable to repair double strand DNA breaks. In fact, this homologous recombination deficiency (HRD) was exploited in what we will explore as a possible evolutionary advantage of having a BRCA mutation. These discoveries ultimately described the strategy employed to find a target for BRCA-associated cancers. ${ }^{13,14}$

While it seemed counterintuitive to target a protein that is responsible for repairing DNA, it was in discovering how this protein promoted cancer cell growth and proliferation that scientists targeted this pathway that ultimately led to cancer cell death. Not only does this lead to a more targeted approach to attacking those cancer cells but also it does so with less systemic toxicity. Attacking this protein, poly-ADP ribose polymerase (PARP), has led to the development of poly-ADP ribose polymerase inhibitors (PARPi). PARPi have not only advanced ovarian cancer therapy but also are being studied in other cancers as well. Initially benefiting patients with BRCA mutations, now PARPi therapy has been shown to be effective in both the treatment and maintenance settings for ovarian cancer with a platinum-sensitive indication. In this review, we will be examining the various targeted treatments of BRCA mutationassociated ovarian cancer with a specific focus on rucaparib and its journey through development and approval in the US.

\section{$B R C A I$ and $B R C A 2$}

The use of PARPi in the setting of ovarian cancer was the first time when targeted therapy was used to take advantage of the synthetic lethality associated with having a BRCA mutation (BRCAm). While this concept of synthetic lethality will be later explored in this paper, a discussion is owed to the genes behind the breast and ovarian cancer syndrome.
Henry Lynch, an American physician, was the first to associate breast and ovarian cancer in the same family. ${ }^{15}$ It took nearly 20 years after that, in 1994, for Mary-Claire King to discover that the gene, $B R C A 1$, on chromosome 17 was associated with breast and ovarian cancers. ${ }^{16,17}$ Several years later, researchers led by Michael Stratton in the UK discovered the second gene, BRCA2, located on chromosome $13 .{ }^{18}$ In looking at all women with epithelial ovarian cancer, approximately $15 \%-20 \%$ carry a predilection to breast and ovarian cancer by harboring a deleterious mutation in either the $B R C A 1$ or $B R C A 2$ gene (BRCA1/2). This risk is increased in women of Ashkenazi Jewish, Icelandic, and French-Canadian descent.

The $B R C A 1 / 2$ genes are tumor suppressor genes, which encode for proteins that repair double-stranded breaks (DSB) in DNA. With a deleterious mutation within these genes, women are predisposed to losing the BRCA1/2 proteins. Inherited in an autosomal dominant fashion, having one mutation does not guarantee that one will develop cancer. It is only when a secondhit or deletion in the homologous gene occurs - termed the two-hit hypothesis - that cancer develops.

The lifetime risk of developing breast cancer with a $B R C A 1 / 2$ genetic mutation is $50 \%-85 \%$. The risk of ovarian cancer with $B R C A 1$ mutation is upwards to $40 \%$ whereas the risk with $B R C A 2$ is $12 \%-20 \%$. While women with BRCA1 mutations tend to develop ovarian cancer in their 30 s and $40 \mathrm{~s}$, those with BRCA2 mutations are often diagnosed in their mid60 s, similar to the age non-mutation carriers are diagnosed. ${ }^{19}$ Risk-reducing gynecologic surgery to prophylactically remove tubes and ovaries greatly decreases the risk of developing carcinomas of the ovary and/or fallopian tube. At the time of prophylactic surgery, occult carcinomas may be present in up to $4 \%$ of women. The risk of ultimately developing primary peritoneal carcinoma is $1 \%-2 \%$ in these patients.

\section{The concept of synthetic lethality}

Although the concept of synthetic lethality is not unique to PARPi, it was the first reported interaction between a tumor suppressor gene and its protein that was described and is displayed in Figure 1. It is also the mechanism exploited by PARPi as its means of providing its therapeutic potential. ${ }^{20}$ The PARP protein binds to DNA that has experienced single stranded breaks. Whether this is because the cancer cell itself has developed those breaks inherently, if there are a large proportion of these breaks because of BRCA deficiency in that tumor, or because a chemotherapeutic agent has caused those errors, PARP binds to those breaks to repair the DNA. This leads to proliferation of that DNA. This is where the role 


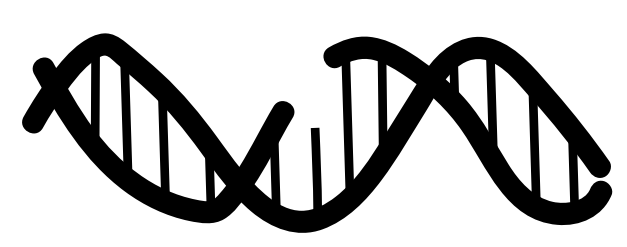

Single strand break (SSB)

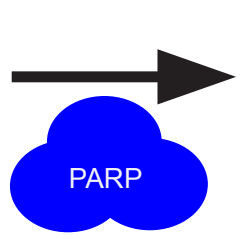

PARP

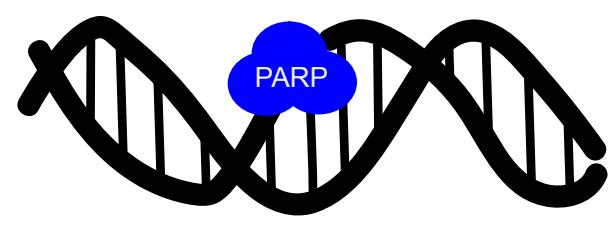

PARP recruited for SSB repair

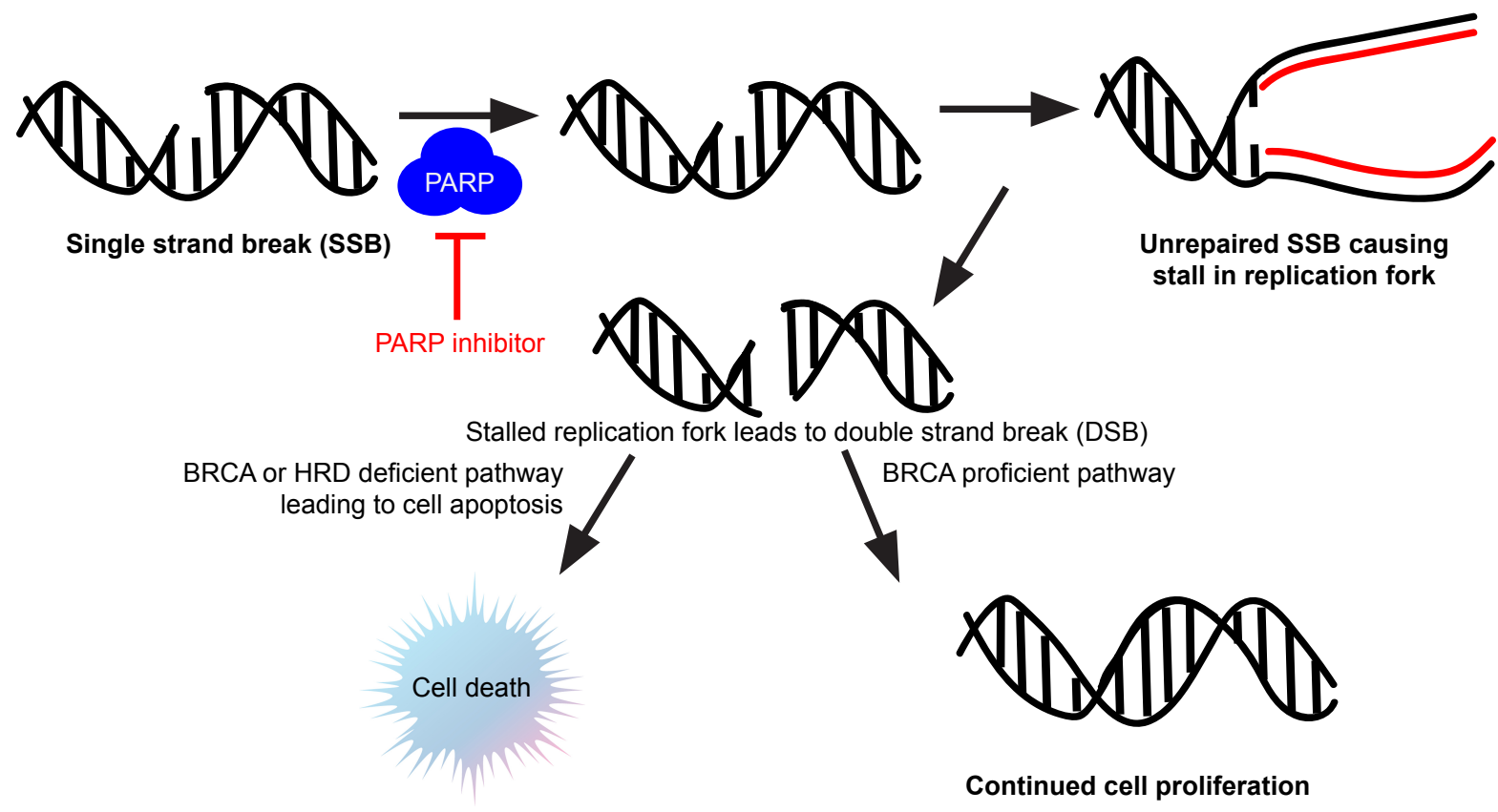

Synthetic lethality

Figure I Concept of synthetic lethality explained with PARP inhibitors in the setting of BRCA mutation.

Abbreviations: DSB, double-stranded break; HRD, homologous recombination deficient; PARP, poly-adenosine diphosphate ribose polymerase; SSB, single-stranded break.

of PARPi has come into play by inhibiting the PARP protein and leading DNA to use the more error-prone pathway of non-homologous end-joining (NHEJ). ${ }^{21}$ This typically leads to cell death and decreased proliferation of those cancer cells. The combination of the BRCA mutation (which is non-lethal) with a synthetic (i.e., pharmacologic) PARPi leads to the synthetically lethal phenotype in cancer cells.

\section{Homologous recombination deficiency}

This concept of synthetic lethality was first reported by American geneticist Calvin Bridges who was studying the non-lethal wingless mutation in fruit flies. HRD was described independently by Farmer et $\mathrm{al}^{13}$ and Bryant et al. ${ }^{14}$ They demonstrated, in vitro, that tumors lacking the HRD pathway were prone to errors in DNA repair during replication. Holiday junctions, named after Robin Holiday who proposed the mechanism of HRD in 1964, occur when homologous chromosomes swap DNA. ${ }^{22}$ Copying the DNA from the opposing strand through branch migration, the DNA gets repaired in this fashion. Given this knowledge about HRD, scientists took advantage of it as the biological mechanism behind PARPi, as it essentially destroyed cells in this error-prone pathway. They also realized that not only did BRCAm carriers exhibit HRD, but also this feature was present in some tumors of patients who were BRCA wild-type (BRCAwt). This led to the development and testing of assays designed to find those tumors with the HRD phenotype.

HRD assays, genetic panels, and the evolution of genetic testing

In June 2013, the Supreme Court ruled against Myriad Genetics' (Myriad Genetics, Inc., Salt Lake City, Utah, USA) exclusive licensing rights to $B R C A 1 / 2$, making it available for other laboratories to develop assays to test for it. Since then, there have been a variety of tumor profiling panels that test blood as well as the tumor itself for various genetic biomarkers. The reason why these tests were developed was because patients who may not have had a mutation (gBRCAmut) were still likely to respond to PARPi because of the tumor's (somatic) deficiency in BRCA1/2 or because of another defect in homologous recombination. 
The first assay developed was myChoice ${ }^{\circledR}$ HRD (Myriad Genetics, Inc.). It tests for three biomarkers: tumor gene loss of heterozygosity ( $\mathrm{LOH}$ ), telomeric allelic imbalance, and largescale state transitions. This assay was tested in NOVA, ${ }^{23}$ the study that tested niraparib maintenance for platinum-sensitive recurrent ovarian cancers, where HRD-positive tumors were considered positive after looking at whether the combination of the three tests produced an algorithmic score of 42 or greater. In looking at the three biomarkers themselves, Swisher et al, in ARIEL2 part 1 found that tumor LOH was a more sensitive predictor to response in BRCA wild-type (BRCAwt) tumors than other homologous recombination genes. ${ }^{11}$ In ARIEL2 part 1 and ARIEL3, the individual tumor tissue samples were tested using Foundation Medicine's T5 next generation sequencing (NGS) assay, FoundationFocus ${ }^{\mathrm{TM}} \mathrm{CD}_{\mathrm{x}}$ BRCA Test, (Cambridge, MA, USA), to detect BRCA mutations within the tumor. The $\mathrm{LOH}$ of $14 \%$ or greater was considered a positive result for HRD based on retrospective analysis of the patients that had statistically significant increased PFS in analyzing The Genome Cancer Atlas. Interestingly, in ARIEL3, the LOH cut-off was based on the examination of ARIEL2 tumor samples and used the slightly higher cut-off of $16 \% .{ }^{12}$ The FDA also approved FoundationFocus ${ }^{\mathrm{TM}} \mathrm{CDx}_{\text {BRCA LOH }}$ (Foundation Medicine, Inc.), which became the first FDA approved companion test paired with rucaparib to test for gBRCAmut, genomic LOH, and HRD status. It was tested in ARIEL3 as a predictive biomarker for rucaparib maintenance therapy.

Although these assays were developed as a way to predict tumor biomarkers that may indicate a patient will be more responsive to certain therapies, they potentially have the ability of providing genetic information to the patient. This may have implications for the patient in relation to their tumor but also exposing what their inherited risk may be to other cancers. As this may have an impact on the individual as well as their family, it is important to determine a patient's readiness and willingness to know and understand her genetic information. Coupling this effort with dedicated genetic counselors may also help provide this additional support to their cancer therapy.

\section{Maintenance therapies in ovarian cancer}

Before discussing trials that brought PARPi and specifically rucaparib FDA approval, the concept of maintenance therapy in ovarian cancer should be discussed preceding the PARPi maintenance trials. As stated above, despite effective frontline therapy that places the majority of women into clinical remission, recurrence rates are as high as $75 \%$. This not only has serious medical consequences but also tends to affect patient psyche. It is also one of the pivotal reasons why effective maintenance therapy is essential for this cancer. Various studies have been conducted to explore maintenance therapy in this disease, however, until recently most have not gained traction until the advent of PARPi.

A Cochrane review examined the various trials in maintenance therapies up to May 2009. ${ }^{24}$ These were using chemotherapeutic agents - platinum-based, doxorubicin, or paclitaxel for maintenance. They concluded that there was no benefit in these maintenance strategies compared to chemotherapy alone. Although not designed to examine the effect of toxicities on discontinuation of the drugs, it is common for these systemic therapies to have adverse effects that not only lead to discontinuation but also greatly affect quality of life.

Pecorelli et al published a phase III RCT of patients who underwent first-line treatment on paclitaxel and platinumbased therapy with complete responses and then were randomized to paclitaxel for maintenance every three weeks or observation. This trial did not find this strategy to be any more effective than observation alone..$^{25}$

Angiogenesis inhibitors have also been studied as maintenance treatments in ovarian cancer. The vascular endothelial growth factor inhibitor, bevacizumab (Genentech, Inc., South San Francisco, CA, USA), has been studied in various cancer types including ovarian cancer. Gynecologic Oncology Group (GOG) protocol 0218 was a phase 3 randomized trial that studied anti-angiogenesis therapy in women with newly diagnosed suboptimally debulked stage III and completely resected stage IV ovarian carcinoma. Following randomization, patients were treated on one of three arms: standard chemotherapy with placebo during initiation and maintenance therapy; chemotherapy with bevacizumab during initiation but placebo during maintenance; and finally, chemotherapy with bevacizumab during initiation and maintenance. Treatment on the bevacizumab-throughout arm was associated with approximately a 4-month improvement in PFS compared to control. This ultimately led to its FDA approval for use as maintenance in ovarian cancer in June 2018. ${ }^{26}$

The next generation of maintenance therapy involves PARPi. On March 27, 2017, the FDA approved niraparib (Tesaro, Inc., Waltham, MA, USA) for maintenance therapy for patients with platinum-sensitive ovarian cancer. Olaparib (AstraZeneca plc, London, UK) was approved on August 17, 2017 in the US. Rucaparib (Clovis Oncology, Boulder, CO, USA), the drug at the focus of this review, was initially approved for $\mathrm{g} / \mathrm{sBRCAm}$ positive patients on December 19, 2016 and most recently with expanded approval as a maintenance therapy for platinum-sensitive patients, on April 6, 2018. Two important points concerning the maintenance therapy labels of all three PARPi are that the approval has 
been granted irrespective of BRCA mutation status and the PARPi are indicated for women who have had a partial or complete response to second-line platinum-based therapy for recurrent platinum-sensitive disease.

Niraparib used the FDA's fast track priority review and breakthrough therapy designation. As stated above, niraparib was approved for patients who not only were gBRCAmut carriers with high grade serous histology epithelial ovarian, primary peritoneal, or fallopian tube cancers, but also those without the mutation who were considered platinumsensitive. Regulatory approval was based on the results of the NOVA trial showing a statistically significant increase in median PFS of gBRCAmut patients receiving the drug vs those on placebo (21 months vs 5.5 months, $P<0.0001) .{ }^{23}$ Meanwhile, for women who were not gBRCAmut carriers, the drug also had efficacy prolonging their survival, 9.3 months vs 3.9 months $(P<0.0001)$.

Shortly following this, the FDA approved olaparib for women with endometrioid or high grade serous histology epithelial ovarian, primary peritoneal, or fallopian tube cancers both from gBRCAmut carriers and also platinum-sensitive patients regardless of their BRCA status. The capsules had been approved three years previously for gBRCAmut ovarian cancer patients fourth line therapy and beyond. When the FDA approved this drug in the maintenance setting, it approved the tablet formulation. The indications were the same as for niraparib, maintenance therapy for recurrent, platinum-sensitive patients regardless of BRCA mutation status. This was based on SOLO2 and Study 19.27,28

Most recently, rucaparib was approved in the maintenance setting, after a priority review of the data from ARIEL3. ${ }^{12}$ This was approved for women with endometrioid or high grade serous histology epithelial ovarian, primary peritoneal, or fallopian tube cancers. This study reported that there was an increased median PFS in patients taking rucaparib who were in the HRD and the sBRCAmut subgroup.

The reason why PARPi are more of a viable maintenance strategy in ovarian cancer patients is threefold: (1) they are orally bioavailable drugs; (2) they tend to be well tolerated with limited toxicities; and (3) they take advantage of the targeted protein that most of these tumors overexpress, PARP. An important point worth mentioning is that although PARPi are effective in both mutation and non-mutation carriers, the drugs work better among those patients with gBRCAmut. There have been no head-to-head trials comparing the three available PARPi in the maintnenance or treatment setting. Ultimately, sensitivity to PARPi is predicated on three biomarkers: molecular signature, platinum-sensitivity, and number of lines of therapy.

\section{Focus on rucaparib}

\section{Early history of PARPi and preclinical studies}

PARPi were initially studied in conjunction with chemotherapy and thought to be chemosensitizers. This was mostly because in preliminary studies they were tested in xenograft models in conjunction with platinum agents. The first PARP inhibitor initially brought to humans was Clovis Oncology's rucaparib, AG014699. This was used in combination with temozolomide in glioblastoma with promising results in a phase I trial. ${ }^{29}$

Preclinical studies demonstrated that rucaparib led to cell death and apoptosis in $B R C A 1 / 2$ mutant or epigeneticallysilenced $B R C A 1 / 2$ cell lines. Mouse xenografts treated with this PARPi also slowed the growth of tumors without $B R C A 1 / 2 .^{30,31}$ Rucaparib had demonstrated a synergistic effect on cell apoptosis when tested in combination with an MDM2-p53 binding antagonist. $P 53$, a gene frequently mutated in high-grade serous ovarian cancers, encodes for a protein that leads to cell cycle regulation. Alone, rucaparib did not stabilize p53 but in combination with the antagonist, it induced stabilization. This suggests that rucaparib does not likely rely on $\mathrm{p} 53$ activation in its cytotoxicity and that the synergistic effect was also not explained by this pathway activation. ${ }^{32}$ Intrinsic fluorescence of rucaparib was noted with an excitation/emission maximum of $355 / 480 \mathrm{~nm}$ and a quantum yield of $0.3 .{ }^{33}$ This was assessed using confocal microscopy and flow cytometry and allowed for drug distribution to be characterized. This study noted that with higher PARP1 expression, nuclear rucaparib uptake increased and had an intracellular half life of 6.4 hours. In addition, rucaparib increased nicotinamide adenosine diphosphate $\left(\mathrm{NAD}^{+}\right)$in a mouse model treated with temozolomide. Temozolomide, known for its effect on $\mathrm{NAD}^{+}$ depletion, was unable to prevent $\mathrm{NAD}^{+}$accumuluation when used in combination with rucaparib. ${ }^{34}$ This demonstrated another biomarker that can be used to assess for rucaparib sensitivity in vivo. This inhibition of $\mathrm{NAD}^{+}$-dependent processes has also been demonstrated in vivo in a mouse xenograft model leading to vasodilation. Tumor vessel perfusion analyses have shown that PARPi led to increased vasodilation in tumor-recruited blood vessels. ${ }^{35,36}$ This may potentiate the response when used in combination with chemotherapy or other cytotoxic agents.

Ultimately, phase I studies established rucaparib as a safe and tolerable drug. ${ }^{37}$ These trials determined that it was both efficacious and manageable from a toxicity standpoint at a 600 mg oral twice-daily dose with activity in patients with platinum-sensitive disease and BRCA1/2 mutations. The adverse events noted were similar to those reported in later phase II/ III studies namely nausea, fatigue, anemia, and elevations in liver enzymes. Recently, phase I studies have also attempted to study it in conjunction with concurrent chemotherapy and 
established that it is safe in combination with chemotherapy but required a lower dose of rucaparib at $240 \mathrm{mg}$ orally daily. ${ }^{38}$

Although phase II/III trials have demonstrated rucaparib's efficacy in multiple cohorts, resistance to this therapy has been observed. This has led to preclinical research looking at what may be inducing this mechanism of resistance. Rucaparib's mechanism of synthetic lethality is primarily based on a cell's deficiency in homologous recombination. Scientists looked at whether restoring homologous recombination in a cell may be leading to resistance to this drug. In fact, in examining the tumor samples from ARIEL2 part 1, secondary mutations in RAD51C and RAD51D, genes associated with HRD, conferred PARP inhibitor resistance. ${ }^{39}$ Conversely, a primary mutation in RAD51C and RAD51D predictably, like that of BRCA1/2 mutation, conferred sensitivity to rucaparib. Similarly, another study exploring non-homologous end-joining (NHEJ), the mechanism by which double-stranded breaks in cells deficient in homologous recombination are repaired, found that cell lines that were NHEJ-deficient actually were resistant to rucaparib. ${ }^{40}$ This is likely due to the lack of synthetic lethality in these cell types. By having DNA repair with an NHEJ-dependent pathway, it is more susceptible to damage by rucaparib.

\section{ARIEL2 part I and Study 10}

The promising results from ARIEL2 part $1^{11}$ and Study $10^{37}$ are ultimately what led to the US FDA's accelerated approval, priority review status, designation as a breakthrough therapy, and orphan drug designation of rucaparib on December 19, 2016 for treatment for recurrent high-grade serous epithelial ovarian, fallopian tube, or primary peritoneal cancer patients with s/gBRCAmut who had been treated with two or more lines of chemotherapies. Additionally, the FDA approved the first next-generation-sequencing (NGS) companion diagnostic assay, FoundationFocus ${ }^{\mathrm{TM}} \mathrm{CD}_{\mathrm{x}}$ BRCA Test, (Foundation Medicine, Inc.) along with rucaparib as was described earlier.

Integrating the results from the efficacy population of Study 10 and ARIEL2 part 1, an objective response rate (ORR) of 53.8\% (95\% CI: 43.8-63.5) was reported with a median duration of response (DOR) of 9.2 months $(95 \% \mathrm{CI}$ : 6.6-11.6). ${ }^{41}$ Study 10 specifically was a combined phase I/II trial to assess disease progression or toxicity, as well as the recommended phase II dose (RP2D) based on the maximum tolerated dose (MTD) from part 1. Part 1 of Study 10 was designed as a dose-escalation study and to evaluate whether rucaparib could be taken with or without food. ARIEL2 was also a two-part, phase II, open label trial to assess for disease progression, unacceptable toxicity or death. ${ }^{11}$ Part 1 was open to patients with platinum-sensitive high-grade ovarian cancer with one prior platinum regimen. Part 2 was open to patients who had been treated with not more than 3 prior chemotherapy regimens, had been treatment free for greater than 6 months, and had measureable, recurrent disease by RECIST. They had to have disease amenable to biopsy, unless they had a known BRCA mutation and the amount of archival formalin-fixed paraffin-embedded (FFPE) tumor tissue was sufficient for analysis. The study designs for Study 10 and ARIEL2 can be found in Figures 2 and 3, respectively. Similar to other PARP-inhibitor trials, exclusion criteria included prior treatment with another PARPi, a history of cancer except those that were curatively treated, symptomatic or untreated central nervous system (CNS) metastases, any gastrointestinal disorder that affected absorption, including but not limited to a duodenal stent that would interfere with absorption of the drug and a hospitalization for a small bowel obstruction in the 3 months prior to the study. Additionally, patients with mild hepatic impairment were excluded.
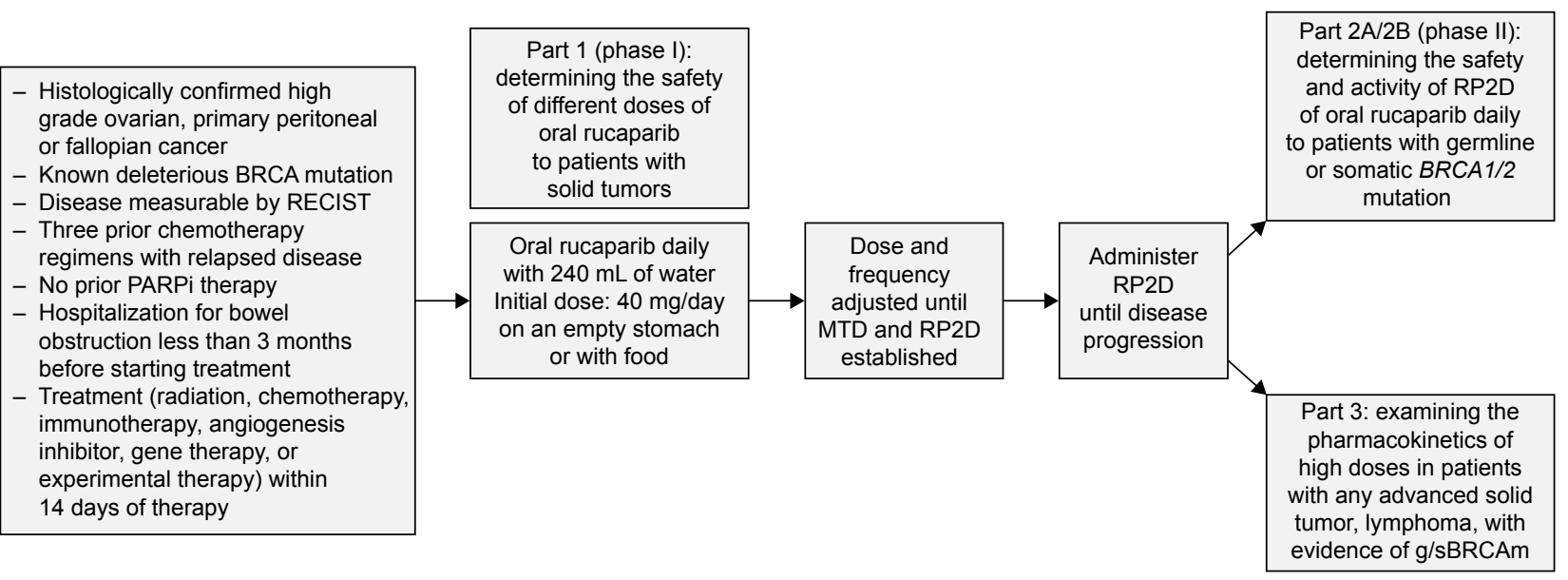

Figure 2 Study 10 design.

Abbreviations: g/sBRCAm, germline/somatic BRCA mutation; MTD, maximum tolerated dose; PARP, poly-adenosine diphosphate ribose polymerase; PARPi, PARP inhibitor; RECIST, response evaluation criteria in solid tumors; RP2D, recommended phase II dose. 
- Recurrent ovarian, primary peritoneal or fallopian cancer

- High grade serous or endometrioid histology

- ECOG performance status 0-1

- No prior PARP inhibitor therapy

- Progression 6 or more months after their most

recent platinum therapy

- Disease measurable by RECIST

- Sufficient archival tumor tissue for analysis
Part 1: primary outcome:

PFS in patients who have received 1 or more platinum-based regimens with platinum-sensitive disease

Figure 3 ARIEL2 study design.

Abbreviations: ECOG, Eastern Cooperative Oncology Group; PARP, poly-adenosine diphosphate ribose polymerase; PFS, progression-free survival; RECIST, response evaluation criteria in solid tumors.

In addition to the primary endpoint assessment based on the integrated efficacy population showing an ORR per Response Evaluation Criteria in Solid Tumors v1.1 (RECIST) $^{41}$ of $53.8 \%$, demonstrating $8.5 \%$ patients with complete response (CR) and $45.3 \%$ with a partial response, it also had a reasonably tolerated safety profile. All patients in the integrated safety population had one treatment-emergent adverse event with the most frequently reported ones including nausea, fatigue, anemia, and elevation in liver enzymes - specifically alanine aminotransferase (ALT) or aspartate aminotransferase (AST). An incidence of myelodysplastic syndrome (MDS) and acute myeloid leukemia (AML) was experienced in one patient each. Notably, neither patient with MDS/AML had a BRCA mutation, but they both had 12 prior cycles of a platinum-based therapy. Ultimately, this tolerable safety profile and efficacy led to the accelerated FDA approval for treatment in this specific BRCAm population.

\section{ARIEL3}

ARIEL3 was a phase III, randomized, double-blind, placebocontrolled trial to study rucaparib maintenance vs placebo in patients with platinum-sensitive, high-grade serous or endometrioid epithelial ovarian, primary peritoneal or fallopian tube cancer. ${ }^{12}$ This study enrolled 564 patients who were randomized in a 2:1 fashion by a computer-generated sequence stratified by their HRD mutation status, PFS interval on their penultimate platinum, and best response to their most recent platinum therapy. Its study design is demonstrated in Figure 4. This trial was conducted at 87 hospitals across 11 countries and designed to evaluate the primary outcome of

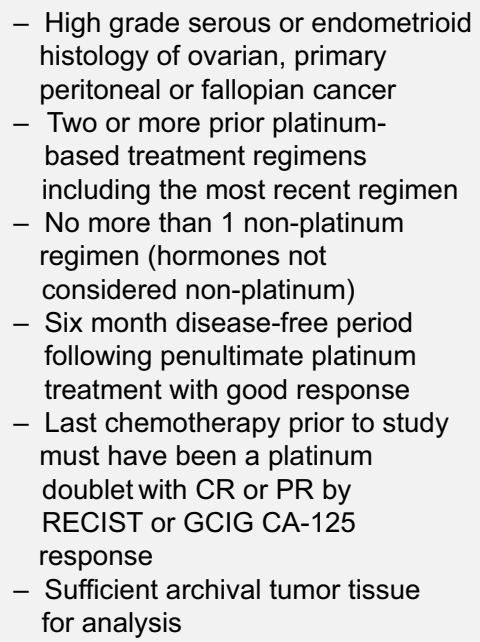

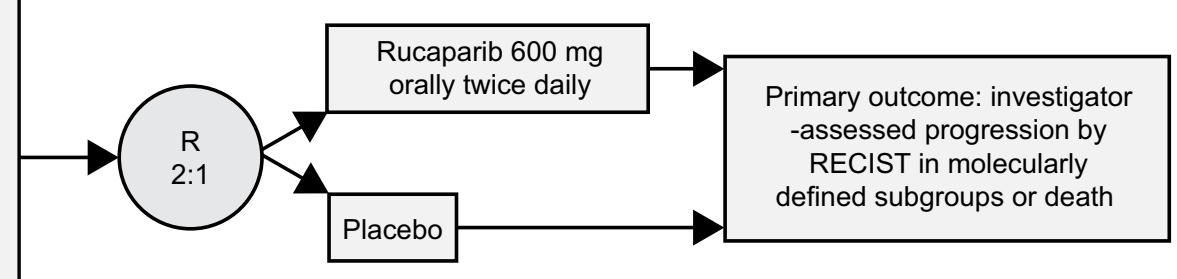

Primary outcome: investigator -assessed progression by defined subgroups or death

Figure 4 ARIEL3 study design.

Abbreviations: CR, complete response; PR, partial response; GCIG, Gynecologic Cancer Intergroup; R, randomization; RECIST, response evaluation criteria in solid tumors. 


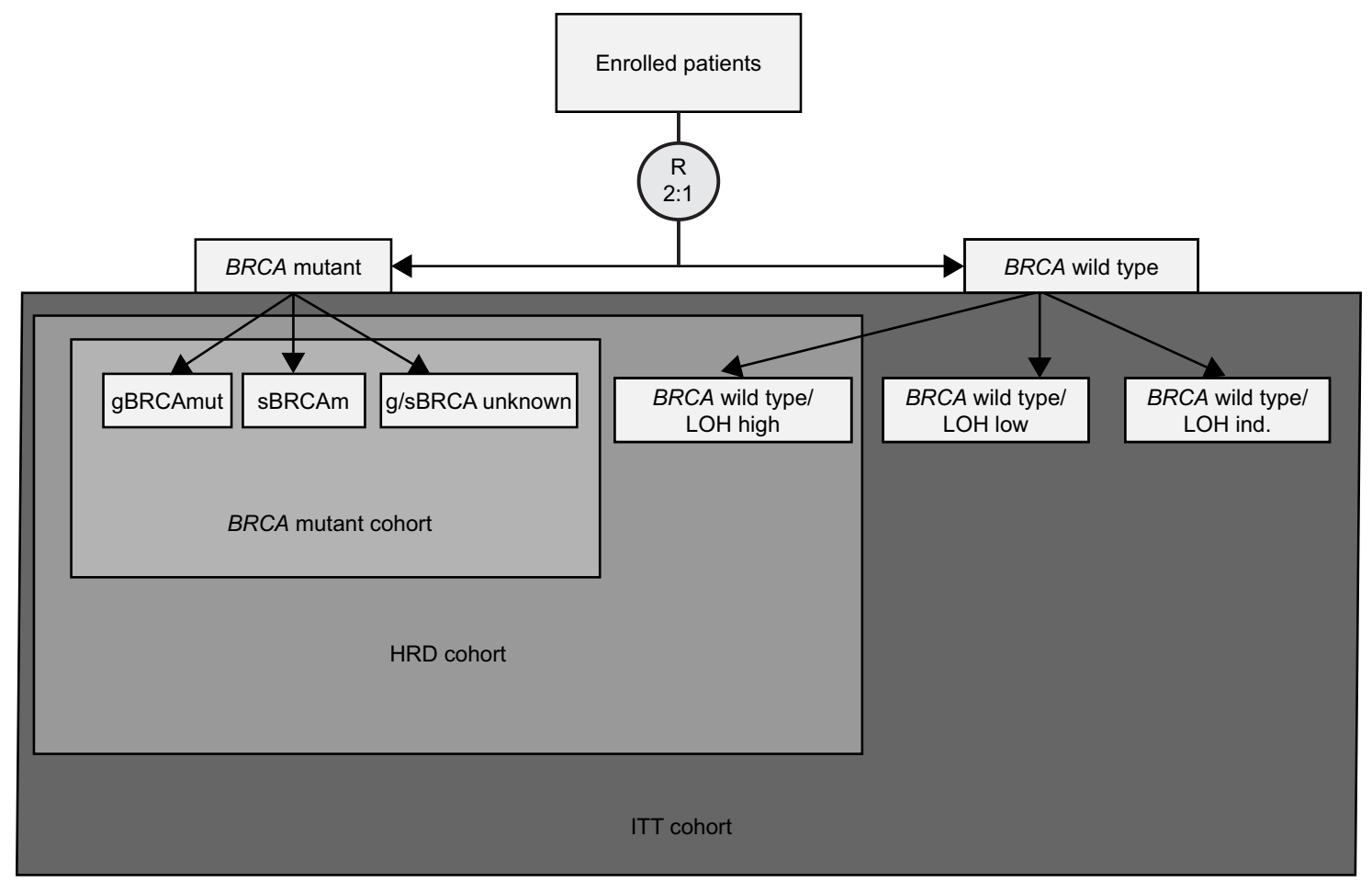

Figure 5 ARIEL3 efficacy analysis nested cohorts.

Abbreviations: gBRCAmut, germline BRCA mutation; HRD, homologous recombination deficiency; ind, indeterminate; ITT, intention-to-treat; R, randomization; sBRCAm, somatic BRCA mutation; LOH, loss-of-heterozygosity.

investigator-determined PFS with an ordered, step-down analyses from three nested cohorts shown in Figure 5. For analyses to continue, statistical significance had to be met in the previous level of analysis. The nested cohorts were patients with BRCAm, HRD tumors (either BRCAm positive or BRCAwt but LOH high), and an intention-to-treat (ITT) subgroup. PFS was assessed every 12 weeks. In all cohorts, rucaparib led to increased PFS: BRCAm 16.6 months (95\% CI: 13.4-22.9) compared to placebo 5.4 months (95\% CI: $3.4-6.7 ; P<0.0001)$, HRD 13.6 months vs 5.4 months in placebo $(P<0.0001)$, ITT 10.8 months vs 5.4 months $(P<0.0001)$. In ARIEL-3, LOHhigh was assigned for tumors with greater than $16 \% \mathrm{LOH}$.

Reportable adverse events included nausea, asthenia, anemia, constipation and vomiting. Grade 3 or greater adverse events occurred more frequently in the rucaparib group (56\%) compared to the placebo group (15\%). Elevations in ALT or AST were noted to be transient and were not associated with other signs of liver toxicity, failure, or elevated bilirubin levels. Elevations in creatinine were also noted and this was attributed to the inhibition of the renal creatinine transporters multidrug and toxin extrusion 1, multidrug and toxin extrusion 2-K, organic cation transport-1 and organic cation transporter which have been inhibited by PARPi including rucaparib. This creatinine elevation also appeared to be transient and occurred relatively early after treatment initiation. Events of MDS/AML were seen in only $1 \%$ of patients $(\mathrm{n}=3): 2$ with BRCA mutations and with 1 without a BRCA mutation who also had an LOH-low tumor.

Ultimately, this favorable adverse event profile and efficacy results led to the accelerated approval of rucaparib in the maintenance setting on April 6, 2018. This approval was granted after the results of ARIEL3 showed that it increased median PFS in all patient subgroups in the cohort who had a CR or PR to their recent platinum-based therapy, with a history of at least two prior regimens. Additionally, it approved the companion test, FoundationFocus ${ }^{\mathrm{TM}} \mathrm{CDx}_{\mathrm{BRCA} \text { LOH }}$ for tumor samples, as described earlier.

\section{Prescribing considerations}

In the last two years, with the FDA approval of three PARPi with expanded indications to be used in maintenance therapy for recurrent platinum-sensitive ovarian cancer, the question has now arisen about which PARPi to prescribe for each patient. All are similarly priced, and each has a patientassistance programs designed to alleviate the financial burden. Furthermore, preliminary cost-effectiveness analyses have not demonstrated maintenance PARP therapy to be a particularly cost-effective strategy. ${ }^{42}$ 
Another consideration to keep in mind when determining which PARPi to use is toxicity. Although most have similar toxicities, severe thrombocytopenia may be doselimiting for niraparib, although this typically is restricted to the first month of therapy, with platelet count recovery to near baseline thereafter. All three of the maintenance PARPi approved are orally bioavailable with only one of the three, niraparib, available in once daily dosing (the other two are twice daily) making ease of administration less of an issue for niraparib. Ultimately, the indications to use one PARPi vs another may be determined by which stage in the disease process the patient is in, whether she is being treated immediately following demonstrable response to second-line platinum-based chemotherapy or is experiencing her third recurrence or beyond. The decision for which PARPi to use must be an individualized one with the patient and oncologist, considering the patient's tumor type, germline and/or somatic BRCA testing as indicated, and previous toxicities while on first-line chemotherapy. As we stated earlier, there are no randomized trials in any disease setting comparing the PARPi with one another.

\section{Rucaparib trials on the horizon}

While there are ongoing trials of rucaparib in various disease types, the two ongoing trials in ovarian cancer are ATHENA (NCT03522246) and ARIEL4 (NCT02855944). These are, respectively, combining it with immunotherapy and comparing its efficacy to current chemotherapy for recurrent disease. In addition to this, phase II/III trials exist for it in prostate, bladder, endometrial, and triple negative breast cancer, which are summarized in Table 1. Importantly, on October 2, 2018, rucaparib was given breakthrough therapy status designation for castration-resistant BRCAm prostate cancer, for those who have received at least one prior androgen receptordirected therapy and taxane chemotherapy.

\section{ATHENA}

Posted to www.ClinicalTrials.gov on May 11, 2018, ATHENA is a phase III, randomized multinational, doubleblinded placebo-controlled, four-arm study of rucaparib with or without the PD-1 inhibitor, nivolumab, following a response to front-line treatment in newly diagnosed patients with stage III or IV ovarian, tubal, or peritoneal carcinoma. Parallel assignment will occur in a randomized fashion to one of four arms: A: oral rucaparib twice daily and IV nivolumab every 4 weeks; B: oral rucaparib and IV placebo; C: oral placebo and IV nivolumab; and D: oral placebo and IV placebo. The primary outcomes of this trial are investigator-assessed
PFS and secondary outcomes are blind-independent central review (BICR) PFS, OS, ORR, and DOR as well as the safety and tolerability of the drug. In addition to including newly diagnosed patients with an ECOG performance status of $0-1$, these patients must have completed cytoreductive surgery (either primary or interval following neoadjuvant chemotherapy) as well as first-line chemotherapy with a response. Tumor tissue is required for analysis and similar to other PARPi/immunotherapy trials, exclusion criteria include known CNS metastases, prior treatment for ovarian cancer beyond first-line therapy, a history of interstitial lung disease or pneumonitis, active known or suspected autoimmune disease, or a condition requiring systemic steroids of greater than $10 \mathrm{mg}$ daily prednisone equivalents. The analysis of this trial will occur in a step-down fashion, similar to ARIEL3. Target enrollment is 1,000 patients.

\section{ARIEL4}

This ongoing trial, currently still recruiting, is a phase III randomized trial of rucaparib vs chemotherapy for BRCA mutation carriers with recurrent ovarian cancer. This is an open-label trial with crossover assignments, designed to enroll about 345 participants. It will evaluate the efficacy of oral rucaparib or an active comparator (platinum-based monotherapy such as cisplatin or carboplatin, a platinum-based doublet such as carboplatin/paclitaxel or carboplatin/gemcitabine, or single agent paclitaxel) as treatment for BRCAm carriers with relapsed ovarian cancer. The active comparator is determined based on local standards and treatment practices. The primary outcome is PFS by RECIST v1.1 with secondary outcomes evaluated including efficacy based on OS as well as the safety and tolerability of rucaparib vs chemotherapy.

\section{Rucaparib with atezolizumab in triple-negative breast cancer or advanced gynecologic cancer}

This study pairs rucaparib with atezolizumab (Genentech, Inc.) a PD-L1 inhibitor, in patients with platinum-sensitive ovarian cancer, previously treated endometrial cancer, or triple-negative breast cancer. This is a phase Ib study investigating the dose, safety, pharmacokinetics and preliminary efficacy of this combination. Part 1 is the dose-finding phase and part 2 is the dose-expansion phase. This is a parallel assignment, open-label trial and will be evaluating adverse events, dose-limiting toxicities, recommended phase II dosing for rucaparib and the number of dose modifications due to adverse events as its primary outcomes. Secondary outcomes include assessing participants for CR or PR by RECIST, ORR, DOR, and PFS. 


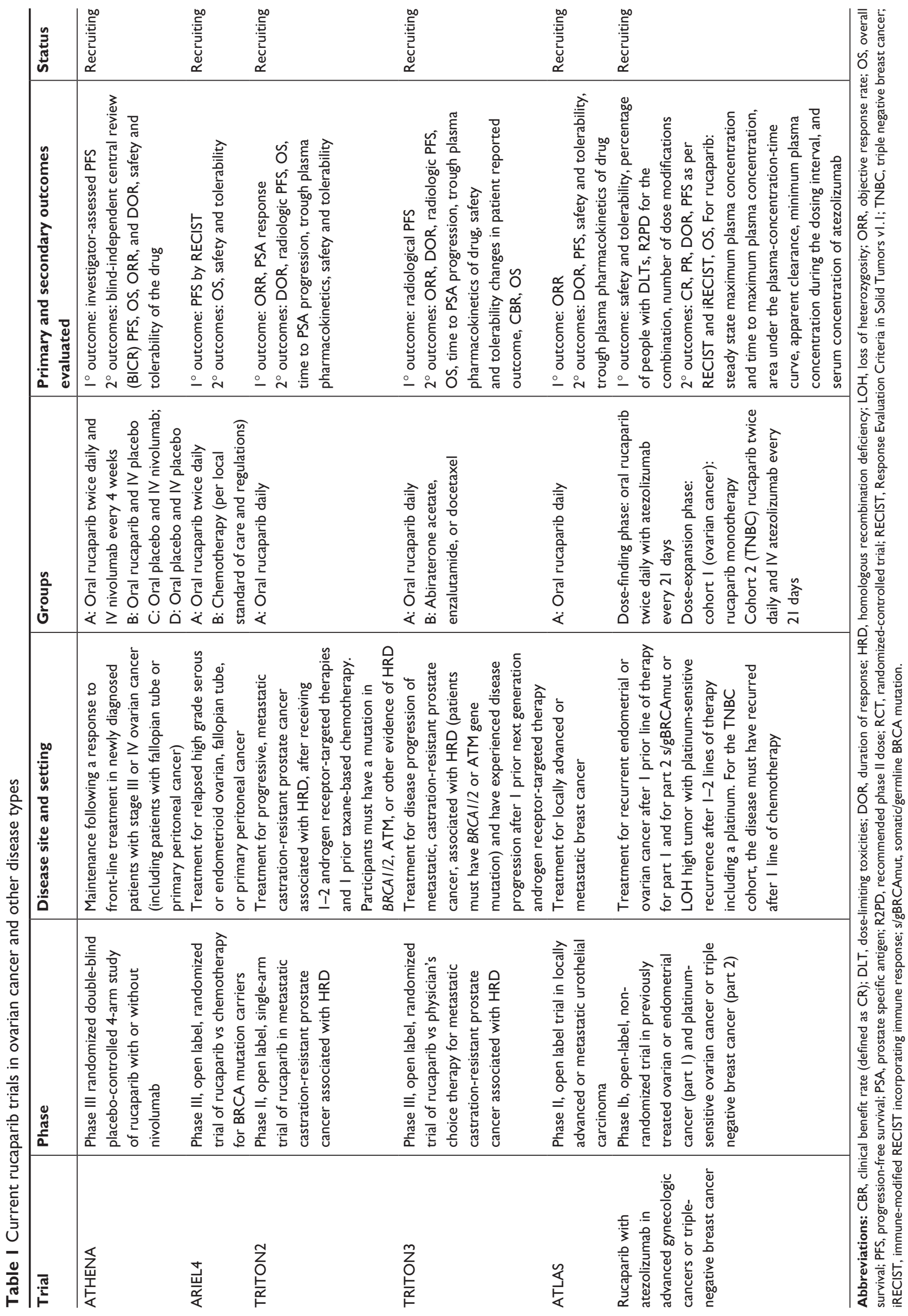


Table 2 Examples of other PARP inhibitors under phase III investigation in the US according to www. ClinicalTrials.gov

\begin{tabular}{|c|c|c|c|}
\hline $\begin{array}{l}\text { PARP } \\
\text { inhibitor }\end{array}$ & Study & $\begin{array}{l}\text { Disease type (ovarian trials } \\
\text { highlighted in bold print) }\end{array}$ & Trial status \\
\hline \multirow[t]{2}{*}{ Iniparib } & $\begin{array}{l}\text { Gemcitabine and carboplatin with or without iniparib (PARPI inhibitor) } \\
\text { in subjects with untreated stage IV NSCLC }\end{array}$ & Stage IV NSCLC, untreated & Completed \\
\hline & $\begin{array}{l}\text { Phase III, multicenter study of gemcitabine/carboplatin with or without } \\
\text { BSI-20I (iniparib) in patients with ER negative, PR negative, HER-2 negative } \\
\text { metastatic breast cancer }\end{array}$ & $\begin{array}{l}\text { Triple-negative, metastatic } \\
\text { breast cancer }\end{array}$ & Completed \\
\hline Talazoparib & $\begin{array}{l}\text { Talazoparib vs physician's choice chemotherapy in advanced or metastatic } \\
\text { breast cancer with BRCA mutation }\end{array}$ & $\begin{array}{l}\text { Advanced or metastatic breast cancer } \\
\text { BRCAm }\end{array}$ & $\begin{array}{l}\text { Active/not } \\
\text { recruiting }\end{array}$ \\
\hline \multirow[t]{4}{*}{ Veliparib } & $\begin{array}{l}\text { Randomized-controlled trial of carboplatin/paclitaxel with or without } \\
\text { veliparib in HER-2 negative or locally advanced unresectable BRCA- } \\
\text { associated breast cancer }\end{array}$ & $\begin{array}{l}\text { Locally advanced breast cancer } \\
\text { BRCAm } \\
\text { HER-2 negative }\end{array}$ & $\begin{array}{l}\text { Active/not } \\
\text { recruiting }\end{array}$ \\
\hline & $\begin{array}{l}\text { Veliparib with carboplatin/paclitaxel and continuation maintenance } \\
\text { in stage III or IV high grade serous epithelial ovarian, fallopian tube, } \\
\text { or primary peritoneal cancer (GOG 3005) }\end{array}$ & $\begin{array}{l}\text { Stage III or stage IV high grade } \\
\text { serous epithelial ovarian, fallopian } \\
\text { tube, or primary peritoneal cancer }\end{array}$ & $\begin{array}{l}\text { Active/not } \\
\text { recruiting }\end{array}$ \\
\hline & $\begin{array}{l}\text { Randomized, double blind, multi-center study comparing veliparib vs } \\
\text { placebo with carboplatin/paclitaxel in previously untreated advanced } \\
\text { or metastatic non-small-cell lung cancer (NSCLC) }\end{array}$ & Stage IV NSCLC, untreated & $\begin{array}{l}\text { Active/not } \\
\text { recruiting }\end{array}$ \\
\hline & $\begin{array}{l}\text { Veliparib with carboplatin/paclitaxel vs physician's choice standard } \\
\text { chemotherapy in patients receiving their first cytotoxic chemotherapy } \\
\text { for metastatic or advanced NSCLC who are current or former smokers }\end{array}$ & $\begin{array}{l}\text { Metastatic or advanced NSCLC } \\
\text { current or former smokers }\end{array}$ & $\begin{array}{l}\text { Active/not } \\
\text { recruiting }\end{array}$ \\
\hline \multirow[t]{3}{*}{ Nirapirib } & $\begin{array}{l}\text { Nirapirib vs placebo in patients with platinum-sensitive ovarian cancer } \\
\text { (NOVA, GOG-30I2) }\end{array}$ & Platinum-sensitive ovarian cancer & Completed \\
\hline & $\begin{array}{l}\text { A phase III trial of nirapirib vs physician's choice chemotherapy in HER-2 } \\
\text { negative, germline BRCA mutation positive breast cancer (BRAVO) }\end{array}$ & $\begin{array}{l}\text { HER-2 negative breast cancer } \\
\text { BRCAm }\end{array}$ & $\begin{array}{l}\text { Active/not } \\
\text { recruiting }\end{array}$ \\
\hline & $\begin{array}{l}\text { Nirapirib vs placebo maintenance treatment in patients with advanced } \\
\text { ovarian cancer following response on front-line platinum-based } \\
\text { chemotherapy (PRIMA) }\end{array}$ & Advanced ovarian cancer & $\begin{array}{l}\text { Active/not } \\
\text { recruiting }\end{array}$ \\
\hline \multirow[t]{5}{*}{ Olaparib } & $\begin{array}{l}\text { Olapirib in BRCA mutation pancreatic cancer patients whose disease has not } \\
\text { progressed on first-line platinum-based chemotherapy (POLO) }\end{array}$ & $\begin{array}{l}\text { Pancreatic cancer } \\
\text { BRCAm }\end{array}$ & Recruiting \\
\hline & $\begin{array}{l}\text { Assessment of efficacy/safety of olaparib mono therapy vs physician's } \\
\text { choice chemotherapy in the treatment of metastatic breast cancer in } \\
\text { patients with germline BRCA //2 mutations (OLYMPIAD) }\end{array}$ & $\begin{array}{l}\text { Metastatic breast cancer } \\
\text { BRCAm }\end{array}$ & $\begin{array}{l}\text { Active/not } \\
\text { recruiting }\end{array}$ \\
\hline & $\begin{array}{l}\text { Cediranib maleate with olapirib or standard chemotherapy in treating } \\
\text { patients with recurrent platinum-resistant or refractory ovarian, fallopian } \\
\text { tube or primary peritoneal cancer }\end{array}$ & $\begin{array}{l}\text { Ovarian, fallopian tube or } \\
\text { primary peritoneal cancer } \\
\text { platinum-resistant or refractory }\end{array}$ & $\begin{array}{l}\text { Suspended } \\
\text { for scheduled } \\
\text { monitoring }\end{array}$ \\
\hline & $\begin{array}{l}\text { Olaparib or cedirinib maleate with olaparib compared with standard } \\
\text { platinum-based chemotherapy in treating patients with recurrent platinum- } \\
\text { sensitive ovarian, fallopian tube, or primary peritoneal cancer }\end{array}$ & $\begin{array}{l}\text { Ovarian, fallopian tube or } \\
\text { primary peritoneal cancer } \\
\text { platinum-sensitive }\end{array}$ & Recruiting \\
\hline & $\begin{array}{l}\text { Platine, Avastin, and Olaparib. A double-blind, phase III trial of Olaparib } \\
\text { vs placebo in patients with advanced stage IIIB-IV high grade serous or } \\
\text { endometrioid ovarian, fallopian ntube, or peritoneal cancer treated with } \\
\text { standard first-line treatment, combining platinum-taxane chemotherapy } \\
\text { and bevacizumab concurrent with chemotherapy and in maintenance }\end{array}$ & $\begin{array}{l}\text { Advanced high grade serous or } \\
\text { endometrioid ovarian, fallopian } \\
\text { tube, or primary peritoneal } \\
\text { cancer }\end{array}$ & Recruiting \\
\hline Pamiparib & $\begin{array}{l}\text { BCB-290 (pamiparib) vs placebo with advanced or inoperable } \\
\text { gastric cancer }\end{array}$ & Advanced or inoperable gastric cancer & Recruiting \\
\hline
\end{tabular}

Abbreviations: ER, estrogen receptor; GOG, Gynecologic Oncology Group; HER-2, human epidermal growth factor receptor 2; HRD, homologous recombination deficient; NSCLC, non-small-cell lung cancer; PR, progesterone receptor.

\section{Ongoing clinical trials in PARPi in multiple cancer types} Some examples of phase 3 randomized trials of PARPi other than rucaparib in ovarian cancer and other tumor types appear in Table 2. Recently published, a 70\% lower risk of disease progression was noted with Olaparib maintenance following primary chemotherapy in BRCA1/2 mutation carriers. ${ }^{43}$

\section{Conclusion}

The future of PARPi in BRCAm carriers as well patients with platinum-sensitive ovarian cancer holds great promise. With the expanded indications approved in rucaparib specifically and similarly for niraparib and olaparib, we would expect more women to receive a PARPi as either a maintenance therapy 
for platinum-sensitive ovarian cancer that has responded to second-line platinum-based therapy, or for third-line or fourthline treatment of BRCA-mutated disease. Unfortunately, as of mid-2018, the on-label uptake of PARPi outside of the clinical trial setting for women suffering with recurrent ovarian cancer is only approximately $20 \%-25 \%$. Perhaps as the labels are expanded to include other tumor types, oncologists will become more familiar with these novel agents and be willing to use them. Although olaparib has the largest footprint of a PARPi in oncology due to its ovarian cancer and recent triplenegative breast cancer indications, rucaparib has the broadest indication in ovarian cancer due to its second-line maintenence indication and treatment indication for $\mathrm{g} / \mathrm{sBRCAmut}$ ovarian cancer for third-line therapy and beyond. A so-called evolutionary advantage of BRCAm was exploited when PARPi were engineered to exploit the mutations in HRD. This synthetic lethality not only proved to benefit BRCAm carriers, but also platinum-sensitive patients irrespective of mutation status. Because tumors harboring BRCA mutations as well as damaging mutations of homologous recombination repair genes are associated with high neoantigen loads, these cancers represent a vulnerable substrate for immunotherapy. Accordingly, the future is likely to bring us novel combinations of the different PARPi with immunologic checkpoint inhibitors.

\section{Disclosure}

Diana Cholakian Pearre, MD, has no disclosures. Krishnansu S. Tewari, MD is on the speakers' bureau for Clovis Oncology, Tesaro, Roche, and Merck. He has contracted research with Genentech, Clovis Oncology, Tesaro, AstraZeneca, CARIS, Regeneron, and Amgen. He also serves on the advisory board of Genmab, Cue, Clovis Oncology, Tesaro, Roche, Merck, Genentech, AstraZeneca, CARIS, Regeneron, and Amgen. The authors report no other conflicts of interest in this work.

\section{References}

1. Noone AM, Howlader N, Krapcho M. SEER Cancer statistics review, 1975-2015. SEER Cancer Statistics Review. Available from: https:// seer.cancer.gov/statfacts/html/ovary.html. Accessed May 21, 2018.

2. Bristow RE, Montz FJ, Lagasse LD, Leuchter RS, Karlan BY. Survival impact of surgical cytoreduction in stage IV epithelial ovarian cancer. Gynecol Oncol. 1999;72(3):278-287.

3. Bristow BRE, Tomacruz RS, Armstrong DK. Maximal cytoreductive surgery for advanced ovarian carcinoma during the platinum era. 2008;20(5):1248-1259.

4. Armstrong D, Plaxe SC, Alvarez RD. Ovarian cancer2. NCCN Clin Pract Guidel Oncol. 2018;2. Available from: https:/www.nccn.org/ professionals/physician_gls/default.aspx\#ovarian. Accessed October 15, 2018.

5. Vergote I, du Bois A, Amant F, Heitz F, Leunen K, Harter P. Neoadjuvant chemotherapy in advanced ovarian cancer: On what do we agree and disagree? Gynecol Oncol. 2013;128(1):6-11.
6. Vergote I, Amant F, Kristensen G, Ehlen T, Reed NS, Casado A. Primary surgery or neoadjuvant chemotherapy followed by interval debulking surgery in advanced ovarian cancer. Eur J Cancer. 2011;47(Suppl 3): S88-S92.

7. Bristow RE, Chi DS. Platinum-based neoadjuvant chemotherapy and interval surgical cytoreduction for advanced ovarian cancer: a metaanalysis. Gynecol Oncol. 2006;103(3):1070-1076.

8. Wagner U, Marth C, Largillier R, et al. Final overall survival results of phase III GCIG CALYPSO trial of pegylated liposomal doxorubicin and carboplatin vs paclitaxel and carboplatin in platinum-sensitive ovarian cancer patients. Br J Cancer. 2012;107(4):588-591.

9. Aghajanian C, Goff B, Nycum LR, Wang YV, Husain A, Blank SV. Final overall survival and safety analysis of OCEANS, a phase 3 trial of chemotherapy with or without bevacizumab in patients with platinumsensitive recurrent ovarian cancer. Gynecol Oncol. 2015;139(1): 10-16.

10. Pujade-Lauraine E, Hilpert F, Weber B, et al. Bevacizumab combined with chemotherapy for platinum-resistant recurrent ovarian cancer: The AURELIA open-label randomized phase III trial. J Clin Oncol. 2014;32(13):1302-1308.

11. Swisher EM, Lin KK, Oza AM, et al. Rucaparib in relapsed, platinumsensitive high-grade ovarian carcinoma (ARIEL2 part 1): an international, multicentre, open-label, phase 2 trial. Lancet Oncol. 2017;18(1): 75-87.

12. Coleman RL, Oza AM, Lorusso D, et al. Rucaparib maintenance treatment for recurrent ovarian carcinoma after response to platinum therapy (ARIEL3): a randomised, double-blind, placebo-controlled, phase 3 trial. Lancet. 2017;390(10106):1949-1961.

13. Farmer H, Mccabe N, Lord CJ, et al. Targeting the DNA repair defect in BRCA mutant cells as a therapeutic strategy. Nature. 2005; 434(7035):917-921.

14. Bryant HE, Schultz N, Thomas HD, et al. Erratum: Specific killing of BRCA2-deficient tumours with inhibitors of poly(ADP-ribose) polymerase. Nature. 2007;447(7142):346-918.

15. Cantor D. The frustrations of families: Henry Lynch, heredity, and cancer control. Med Hist. 2006;50(3):279-302.

16. King MC, Marks JH, Mandell JB, New York Breast Cancer Study Group. Breast and ovarian cancer risks due to inherited mutations in BRCA1 and BRCA2. Science. 2003;302(5645):643-646.

17. Hall JM, Lee MK, Newman B, et al. Linkage of early-onset familial breast cancer to chromosome 17q21. Science. 1990;250(4988): 1684-1689.

18. Wooster R, Bignell G, Lancaster J, et al. Identification of the breast cancer susceptibility gene BRCA2. Nature. 1995;378(6559):789-792.

19. Lu K, Kauff N, Powell B. ACOG practice bulletin 103: Hereditary breast and ovarian cancer syndrome. Obstet Gynecol. 2009;114(106): 192-202.

20. Javle M, Curtin NJ. The role of PARP in DNA repair and its therapeutic exploitation. Br J Cancer. 2011;105(8):1114-1122.

21. Helleday T. The underlying mechanism for the PARP and BRCA synthetic lethality: clearing up the misunderstandings. Mol Oncol. 2011; 5(4):387-393.

22. Holliday R. A mechanism for gene conversion in fungi. Genet Res. 1964;5(2):282-304.

23. Mirza MR, Monk BJ, Herrstedt J, et al. Niraparib maintenance therapy in platinum-sensitive, recurrent ovarian cancer. $N$ Engl J Med. 2016; 375(22):2154-2164.

24. Mei L, Chen H, Wei DM. Maintenance chemotherapy for ovarian cancer. Cochrane Database Syst Rev. 2013;27(28):CD007414.

25. Pecorelli S, Favalli G, Gadducci A, et al. Phase III trial of observation versus six courses of paclitaxel in patients with advanced epithelial ovarian cancer in complete response after six courses of paclitaxel/ platinum-based chemotherapy: final results of the after-6 protocol 1 . J Clin Oncol. 2009;27(28):4642-4648. 
26. Burger RA, Brady MF, Bookman MA, et al. Incorporation of bevacizumab in the primary treatment of ovarian cancer. N Engl J Med. 2011; 365(26):2473-2483.

27. Gourley C, Friedlander M, Matulonis UA, et al. Clinically significant long-term maintenance treatment with olaparib in patients (pts) with platinum-sensitive relapsed serous ovarian cancer (PSR SOC). J Clin Oncol. 2017;35(15 Suppl):5533.

28. Pujade-Lauraine E, Ledermann JA, Selle F, et al. Olaparib tablets as maintenance therapy in patients with platinum-sensitive, relapsed ovarian cancer and a BRCA1/2 mutation (SOLO2/ENGOT-Ov21): a double-blind, randomised, placebo-controlled, phase 3 trial. Lancet Oncol. 2017;18(9):1274-1284.

29. Plummer R, Middleton M, Wilson R, et al. First in human phase I trial of the PARP inhibitor AG-014699 with temozolomide (TMZ) in patients (pts) with advanced solid tumors. J Clin Oncol. 2005; 23(16 Suppl):3065.

30. Drew Y, Mulligan EA, Vong WT, et al. Therapeutic potential of poly(ADP-ribose) polymerase inhibitor AG014699 in human cancers with mutated or methylated BRCA1 or BRCA2. J Natl Cancer Inst. 2011; 103(4):334-346.

31. Ihnen M, Zu Eulenburg C, Kolarova T, et al. Therapeutic potential of the poly(ADP-ribose) polymerase inhibitor rucaparib for the treatment of sporadic human ovarian cancer. Mol Cancer Ther. 2013;12(6): 1002-1015.

32. Zanjirband M, Curtin N, Edmondson RJ, Lunec J. Combination treatment with rucaparib (Rubraca) and MDM2 inhibitors, Nutlin-3 and RG7388, has synergistic and dose reduction potential in ovarian cancer. Oncotarget. 2017;8(41):69779-69796.

33. Kossatz S, Carney B, Farley C, Weber WA, Drain CM, Reiner T. Direct imaging of drug distribution and target engagement of the PARP inhibitor rucaparib. J Nucl Med. 2018;59(8):1316-1320.

34. Almeida GS, Bawn CM, Galler M, et al. PARP inhibitor rucaparib induces changes in NAD levels in cells and liver tissues as assessed by MRS. NMR Biomed. 2017;30(9):e3736-e3738.
35. Mccrudden CM, O'Rourke MG, Cherry KE, et al. Vasoactivity of rucaparib, a PARP-1 inhibitor, is a complex process that involves myosin light chain kinase, P2 receptors, and PARP itself. PLoS One. 2015; 10(2):e0118187.

36. Ali M, Telfer BA, Mccrudden C, et al. Vasoactivity of AG014699, a clinically active small molecule inhibitor of poly(ADP-ribose) polymerase: a contributory factor to chemopotentiation in vivo? Clin Cancer Res. 2009;15(19):6106-6112.

37. Kristeleit R, Shapiro GI, Burris HA, et al. A phase I-II study of the oral PARP inhibitor rucaparib in patients with germline $B R C A 1 / 2$-mutated ovarian carcinoma or other solid tumors. Clin Cancer Res. 2017; 23(15):4095-4106.

38. Wilson RH, Evans TJ, Middleton MR, et al. A phase I study of intravenous and oral rucaparib in combination with chemotherapy in patients with advanced solid tumours. Br J Cancer. 2017;116(7):884-892.

39. Kondrashova O, Nguyen M, Shield-Artin K, et al. Secondary somatic mutations restoring $R A D 51 C$ and $R A D 51 D$ associated with acquired resistance to the PARP inhibitor rucaparib in high-grade ovarian carcinoma. Cancer Discov. 2017;7(9):984-998.

40. Mccormick A, Donoghue P, Dixon M, et al. Ovarian cancers harbor defects in nonhomologous end joining resulting in resistance to rucaparib. Clin Cancer Res. 2017;23(8):2050-2060.

41. Oza AM, Tinker AV, Oaknin A. Antitumor activity and safety of the PARP inhibitor rucaparib in patients with high-grade ovarian carcinoma and a germline or somatic BRCA1 or BRCA2 mutation: Integrated analysis of data from Study 10 and ARIEL2. Gynecol Oncol. 2017;147(2):267-275.

42. Liu AY, Cohen JG, Walsh CS, Holschneider CH, Sinno AK. A costeffectiveness analysis of three PARP inhibitors for maintenance therapy in platinum-sensitive recurrent ovarian cancer. Gynecol Oncol. 2017;147(1):196.

43. Moore K, Colombo N, Scambia G, et al. Maintenance olaparib in patients with newly diagnosed advanced ovarian cancer. $N$ Engl J Med. Epub 2018 Oct 21
Therapeutics and Clinical Risk Management

\section{Publish your work in this journal}

Therapeutics and Clinical Risk Management is an international, peerreviewed journal of clinical therapeutics and risk management, focusing on concise rapid reporting of clinical studies in all therapeutic areas, outcomes, safety, and programs for the effective, safe, and sustained use of medicines. This journal is indexed on PubMed Central, CAS,

\section{Dovepress}

EMBase, Scopus and the Elsevier Bibliographic databases. The manuscript management system is completely online and includes a very quick and fair peer-review system, which is all easy to use. Visit http://www.dovepress.com/testimonials.php to read real quotes from published authors. 